\title{
Transferability of barley EST markers used for analysis T. aestivum - H. marinum subsp. gussoneanum introgression lines
}

\author{
N.V. Trubacheeva ${ }^{1 *}$, E.D. Badaeva ${ }^{2}$, T.S. Osadchaya ${ }^{1}$, L.A. Pershina ${ }^{1}$ \\ ${ }^{1}$ Institute of Cytology and Genetics, SB RAS, Novosibirsk, Russia \\ ${ }^{2}$ Vavilov Institute of General Genetics, Moscow, Russia
}

DOI 10.18699/ICG-PlantGen2019-63

(c) Autors, 2019

* e-mail: ntr@ngs.ru

\begin{abstract}
We evaluated the applicability of seventy-eight $H$. vulgare EST markers for studying bread wheat- $H$. marinum subsp. gussoneanum substitution and addition lines. Of all the markers studied, thirty-six (46\%) were amplified in H. marinum ssp. gussoneanum and wheat introgression lines. The identification of wild barley chromosomes using EST markers confirmed the GISH and C-banding data. Thus, it was established that the H. vulgare EST markers can be successfully used to identify the chromosomes of $H$. marinum subsp. gussoneanum in introgression lines of wheat.
\end{abstract}

Key words: EST markers; introgression alloplasmic lines; wild barkey.

\section{Introduction}

Wild relatives have been employed successfully in common wheat breeding programmes to introgress agronomically important genes (Ceoloni et al., 2014). Sea barley (Hordeum marinum subsp. gussoneanum, $2 n=28$ ) has potentially useful traits such as resistance to abiotic stresses, including high salt tolerance (Garthwaite et al., 2005), waterlogging (Garthwaite et al., 2005), and tolerance to combined salinity and waterlogging resulting in low $\mathrm{O}_{2}$ concentrations (Malik et al., 2009). These resistance traits may have been transferred to wheat due to the crossability of wild barley with bread wheat. Chromosomes from $H$. marinum could be introduced into common wheat through wheat-barley hybrids and backcrossing to wheat (Pershina et al., 2009; Trubacheeva et al., 2009). Introgressed segments can be assessed by in situ hybridisation, which readily distinguishes $H$. marinum chromosomes from those of wheat (Trubacheeva et al., 2009). Molecular markers capable of detecting small segments of H. marinum chromatin in a wheat background would also enhance the use of this wild species to increase wheat genetic resources. The aim of this work was to study the amplification of EST markers of barley $H$. vulgare in the genome of the wild barley $H$. marinum ssp. gussoneanum and to assess their use for detecting barley chromatin segments in the alloplasmic bread wheat-H. marinum subsp. gussoneanum introgression lines.

\section{Materials and methods}

The accessions of the barley $H$. marinum ssp. gussoneanum Hudson $(2 n=4 x=28), H$. vulgare cv. 'Nepolegaushii' $(2 n=$ $2 x=14$ ), and bread wheat $T$. aestivum cv. 'Pyrotrix 28 ' were used for the initial transferability analysis of 78 EST markers. Alloplasmic (with the cytoplasm of $H$. marinum) wheat-barley introgression lines were studied using GISH, C-banding and EST analysis. A set of 78 EST-SSR markers developed by Hagras et al. (2005) and uniformly distributed across the $H$. vulgare chromosomes were tested for amplification of $H$. marinum ssp. gussoneanum DNA. PCR conditions followed a touch-down protocol as described by Hagras et al. (2005). Amplified products were separated in $1.5 \%$ agarose gels, stained with ethidium bromide and photographed in ultraviolet light.

\section{Results and discussion}

The transferability of $85 \mathrm{H}$. vulgare EST markers, which were from all the seven homoeologous groups of barley, to the chromosomes of $H$. marinum ssp. gussoneanum was examined. Forty-two markers, i. e., $49 \%$, did not amplify fragments in H. marinum ssp. gussoneanum. Two markers were not polymorphic between $H$. vulgare, $H$. marinum ssp. gussoneanum and T. aestivum and therefore could not be used. These 44 markers were not applicable for analysing lines with chromosomes of $H$. marinum ssp. gussoneanum. Forty-one EST markers, i.e., 48\%, showed a clear single band of the same size in $H$. vulgare and $H$. marinum ssp. gussoneanum but failed to amplify or amplified a fragment of different sizes in wheat. Thus, these 41 EST markers of $H$. vulgare were transferable to $H$. marinum ssp. gussoneanum and would be useful in identifying $H$. marinum ssp. gussoneanum chromosomes in bread wheat backgrounds.

The presence of barley chromosomes in the alloplasmic lines was detected using GISH analysis and C-banding of chromosomes. GISH was performed to reveal the chromosome configuration and the presence of $H$. marinum ssp. gussoneanum chromosomes in alloplasmic bread wheat- $H$. marinum subsp. gussoneanum lines. It was established that all the lines studied carry H. marinum ssp. gussoneanum chromosomes and are either substitution or addition lines. We found a disomic substitution line with $2 n=40 \mathrm{w}+2 \mathrm{H}^{\text {mar }}$, a ditelosomic addition line with $2 n=42 \mathrm{w}+2 \mathrm{tH}^{\mathrm{mar}}$ and a disomic addition line with $2 n=42 \mathrm{w}+2 \mathrm{H}^{\operatorname{mar}}$ (Figure 1, a). In the line derived from incomplete amphiploid $(2 n=54), 12$ wild barley chromosomes were added to 42 wheat chromosomes $\left(42 \mathrm{w}+12 \mathrm{H}^{\mathrm{mar}}\right)$ (Figure 1, $b$ ). Two lines were multiple addition lines carrying two pairs of $H$. marinum ssp. gussoneanum chromosomes $\left(2 n=42 \mathrm{w}+4 \mathrm{H}^{\mathrm{mar}}\right)$ and one line was a multiple substitution line $\left(2 n=36 \mathrm{w}+6 \mathrm{H}^{\mathrm{mar}}\right)$. C-banding confirmed the number of chromosomes in the lines studied and determined the types of substitutions in the alloplasmic wheat-barley substitution lines. 

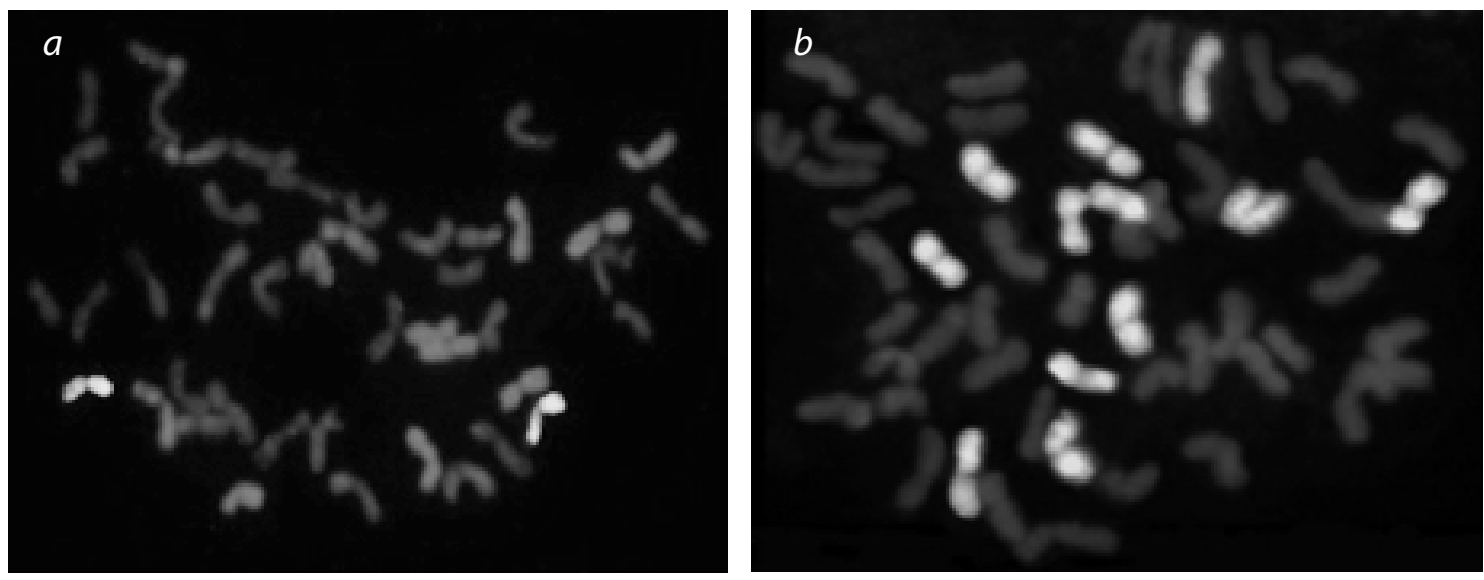

Figure 1. Genomic in situ hybridization with $H$. marinum ssp. gussoneanum genomic DNA (green) probes to mitotic metaphase chromosomes.

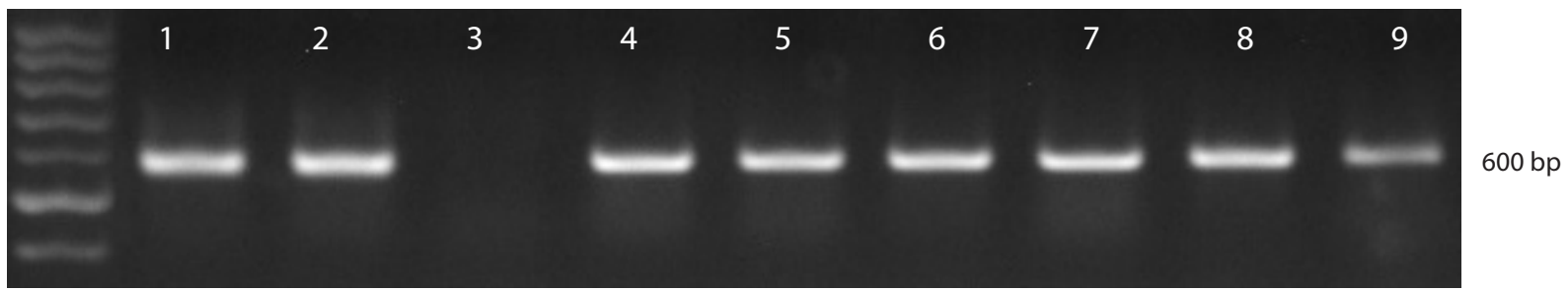

Figure 2. Example of a PCR amplification profile used for identifying chromosome $7 \mathrm{H}^{\text {mars }}$ with EST marker k4573. 1, $H$. vulgare; 2, H. marinum ssp. gussoneanum; 3, T. aestivum; 4, 7H $\mathrm{H}^{\operatorname{mar}}$ (7D) disomic substitution line; $5,7 \mathrm{H}^{\text {mar }}$ (7B) disomic substitution line; $6,1 \mathrm{H}^{\operatorname{mar}}(1 \mathrm{~B}), 5 \mathrm{H}^{\mathrm{mar}}(5 \mathrm{D}), 7 \mathrm{H}^{\operatorname{mar}}(7 \mathrm{D})$ multiple substitution line; $7,7 \mathrm{H}^{\operatorname{mar} S}$ ditelosomic addition line; $8,7 \mathrm{H}^{\mathrm{mar}}$ disomic addition line; 9, $1 \mathrm{H}^{\mathrm{mar}}+7 \mathrm{H}^{\text {mar }}$ multiple addition line.

The following types of substitutions have been identified: $7 \mathrm{H}^{\mathrm{mar}} \mathrm{L}(7 \mathrm{D}), 7 \mathrm{H}^{\mathrm{mar}}(7 \mathrm{D}), 7 \mathrm{H}^{\mathrm{mar}}(7 \mathrm{~B})$, and one line contained three chromosome substitutions, $1 \mathrm{H}^{\operatorname{mar}}(1 \mathrm{~B}), 5 \mathrm{H}^{\mathrm{mar}}(5 \mathrm{D})$ and $7 \mathrm{H}^{\mathrm{mar}}(7 \mathrm{D})$. We identified the addition lines with a pair of telocentric chromosomes for the long arm of chromosome $7 \mathrm{H}^{\mathrm{mar}}$, a pair of telocentric chromosomes for the short arm of $7 \mathrm{H}^{\mathrm{mar}}$ and the line with 42 chromosomes of wheat and a pair of chromosomes $7 \mathrm{H}^{\mathrm{mar}}$.

There is a lack of molecular markers for wild species such as H. marinum subsp. gussoneanum, and the transfer of markers to them from related crop species is a feasible method for genetic analysis (Hagras et al., 2005). Therefore, EST markers of $H$. vulgare were used because cultivated barley is a closely related species of wild barley. The transferable EST markers of all chromosomes were amplified, except for $5 \mathrm{H}^{\mathrm{mar}}$, in the line with $42 \mathrm{w}+12 \mathrm{H}^{\mathrm{mar}}$. All markers specific for $H$. vulgare chromosomes $1 \mathrm{H}, 5 \mathrm{H}$ and $7 \mathrm{H}$ were successfully amplified in the wheat-barley substitution line with three wild barley chromosomes, $1 \mathrm{H}^{\mathrm{mar}}, 5 \mathrm{H}^{\mathrm{mar}}$, and $7 \mathrm{H}^{\text {mar. }}$ In the lines that were disomic for the $7 \mathrm{H}^{\operatorname{mar}}(7 \mathrm{D})$ and $7 \mathrm{H}^{\operatorname{mar}}(7 \mathrm{~B})$ substitutions, as well as in the addition line for $7 \mathrm{H}^{\mathrm{mar}}$, five markers for $7 \mathrm{H}$ were amplified (Figure 2).

In the line with chromosomes $1 \mathrm{H}^{\mathrm{mar}}$ and $4 \mathrm{H}^{\text {mar }}$ and with chromosomes $1 \mathrm{H}^{\mathrm{mar}}$ and $7 \mathrm{H}^{\mathrm{mar}}$, markers located in the homoeologous chromosomes of $H$. vulgare were also amplified. Thus, according to the results of EST analysis, transferable markers of chromosomes $1 \mathrm{H}, 4 \mathrm{H}, 5 \mathrm{H}$ and $7 \mathrm{H}$ are localized on the homoeologous chromosomes of wild barley H. marinum. Localization of EST markers on homoeologous chromosomes in related species was also demonstrated in (Hagras, 2005), where it was found that $90 \%$ of the studied EST markers of cultivated barley are localized on homoeologous $H$. chilense chromosomes. The authors explain this by the fact that EST sequences have a unique character in the genome and are highly conserved. At the same time, the absence of amplification products in $H$. marinum ssp. gussoneanum with $54 \%$ of the $H$. vulgare markers used indicates that the genomes of the two barley species have undergone significant changes in evolution. These two species of barley are known to be phylogenetically distant and belong to different subgenera of the genus Hordeum (Blattner 2015).

\section{Conclusion}

In our work, a combination of cytogenetic and molecular genetic approaches were used for characterization of bread wheat-H. marinum ssp. gussoneanum introgression lines. The results showed that transferable $H$. vulgare EST markers can be successfully used to identify the chromosomes of $\mathrm{H}$. marinum ssp. gussoneanum.

\section{References}

Blattner F.R. Progress in phylogenetic analysis and a new infrageneric classification of the barley genus Hordeum (Poaceae: Triticeae). Breed Sci. 2009;59:471-480. 
Ceoloni C., Kuzmanovic L., Forte P., Gennaro A., Bitti A. Targeted exploitation of gene pools of alien Triticeae species for sustainable and multi-faceted improvement of the durum wheat crop. Crop Pasture Sci. 2014;65:96-111.

Garthwaite A.J., von Bothmer R., Colmer T.D. Salt tolerance in wild Hordeum species is associated with restricted entry of $\mathrm{Na}+$ and $\mathrm{Cl}-$ into the shoots. $J$ Exp Bot. 2005;56(419):2365-2378.

Hagras A.A., Kishii M., Sato K., Tanaka H., Tsujimoto H. Extended application of barley EST markers for the analysis of alien chromosomes added to wheat genetic background. Breed Sci. 2005;55: 335-341.

Malik A.I., English J.P., Colmer T.D. Tolerance of Hordeum marinum accessions to $\mathrm{O} 2$ deficiency, salinity and these stresses combined. Ann Bot. 2009;103(2):237-248. DOI 10.1093/aob/men142.
Pershina L.A., Devyatkina E.P., Belova L.I., Trubacheeva N.V., Arbuzova V.S., Kravtsova L.A. Features of alloplasmic wheat-barley substitution and addition lines (Hordeum marinum subsp. gussoneanum)-Triticum aestivum. Russian J Gen. 2009;45:1223-1229.

Trubacheeva N.V., Efremova T.T., Badaeva E.D., Kravtsova L.A., Belova L.I., Devyatkina E.P., Pershina L.A. Production of alloplasmic and euplasmic wheat-barley ditelosomic substitution lines $7 \mathrm{H}^{1} \operatorname{Lmar}(7 \mathrm{D})$ and analysis of the $18 \mathrm{~S} / 5 \mathrm{~S}$ mitochondrial repeat in these lines. Russ. J. Genet. 2009;45:1438-1443.

Acknowledgements. The work is supported by project No. 03242019-0039, RFBR grant No. 17-04-01738.

Conflict of interest. The authors declare no conflict of interest. 